\title{
Romosozumab efficacy on fracture outcomes is greater in patients at high baseline fracture risk: a post hoc analysis of the first year of the frame study
}

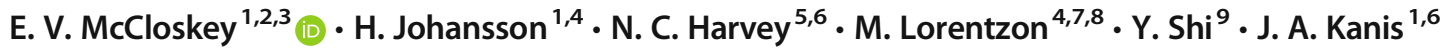

Received: 3 September 2020 / Accepted: 30 December 2020 / Published online: 3 February 2021

(C) The Author(s) 2021

\begin{abstract}
Summary This study aimed to determine the interaction between baseline FRAX ${ }^{\circledR}$ fracture probability and romosozumab efficacy. Using an ITT approach, it was determined that the efficacy of romosozumab on clinical fracture, osteoporotic fracture, and major osteoporotic fracture is significantly greater in patients at high baseline fracture risk, when compared with placebo. Introduction Post hoc analyses of placebo-controlled osteoporosis treatment studies have shown significantly greater reductions of fracture incidence for higher fracture risk patients. This study determined the interaction between baseline FRAX ${ }^{\circledR}$ fracture probability and romosozumab efficacy in the placebo-controlled first year of the phase 3 FRAME study (NCT01575834).

Methods Using an ITT approach, an extension of Poisson regression analysis studied the relationship between treatment, FRAX $^{\circledR} 10$-year probability of major osteoporotic fracture (MOF, calculated without BMD) and risk of first incident fracture (adjusting for age and follow-up time). Treatment interactions considered outcomes of all clinical fractures, osteoporotic fractures, MOF, clinical vertebral fractures, and morphometric vertebral fractures. Two-sided $p$ value of $<0.1$ for the interaction between treatment and FRAX ${ }^{\circledR}$ was considered significant.

Results Compared with placebo, romosozumab reduced the incidence of all fracture outcomes in the first year (range: $32 \%$ reduction in MOF $[p=0.07]$ to $80 \%$ reduction in clinical vertebral fractures $[p=0.038])$. Significant interactions were observed between efficacy and baseline FRAX ${ }^{\circledR}$ probability for composite outcomes of clinical fractures, osteoporotic fractures, and MOF $(p=0.064-0.084)$, but not vertebral fractures $(p>0.3)$. For example, romosozumab decreased all clinical fractures by $22 \%$ at the 25 th centile of FRAX ${ }^{\circledR}$ probability but the reduction was $41 \%$ at the 75 th centile. Exclusion of vertebral fractures from each composite fracture outcome (i.e. only nonvertebral fractures included) showed even stronger interactions with baseline FRAX ${ }^{\circledR}$ probability $(p=0.036-0.046)$.
\end{abstract}

Conclusions Efficacy of romosozumab on clinical fracture, osteoporotic fracture, and MOF is significantly greater in patients at high baseline fracture risk compared with placebo.

Keywords Fracture reduction $\cdot$ Fracture risk $\cdot$ FRAX $\cdot$ Romosozumab

E. V. McCloskey

e.v.mccloskey@sheffield.ac.uk

1 Centre for Metabolic Bone Diseases, University of Sheffield, Sheffield, UK

2 Centre for Integrated Research in Musculoskeletal Ageing (CIMA), Mellanby Centre for Bone Research, University of Sheffield, Sheffield, UK

3 Metabolic Bone Centre, Northern General Hospital, Herries Road, Sheffield S5 7AU, UK

4 Mary McKillop Institute for Health Research, Australian Catholic University, Melbourne, Australia
5 MRC Lifecourse Epidemiology Unit, University of Southampton, Southampton, UK

6 NIHR Southampton Biomedical Research Centre, University of Southampton and University Hospital Southampton NHS Foundation Trust, Southampton, UK

7 Geriatric Medicine, Department of Internal Medicine and Clinical Nutrition, Institute of Medicine, University of Gothenburg, Gothenburg, Sweden

8 Region Västra Götaland, Geriatric Medicine, Sahlgrenska University Hospital, Mölndal, Sweden

9 Amgen Inc, Thousand Oaks, CA, USA 


\section{Introduction}

Romosozumab, a monoclonal antibody that binds and inhibits sclerostin (a negative regulator of bone formation secreted by osteocytes), has a dual effect of increasing bone formation and decreasing bone resorption $[1,2]$. In two large phase 3 clinical trials, romosozumab has proved superior to placebo and oral alendronate in its ability to reduce fractures [3-6]. In the FRAME study (NCT01575834), a pivotal fracture study in postmenopausal women with osteoporosis, a year of romosozumab, $210 \mathrm{mg}$ subcutaneously (s.c.) monthly, followed by a year of denosumab (60 mg s.c. every 6 months) was compared to a year of placebo monthly injections followed by a year of denosumab [3]. The romosozumab arm showed a $73 \%$ lower relative risk of vertebral fractures and a $36 \%$ lower risk of clinical fractures, by the 1-year time point, comparable to the $75 \%$ and $33 \%$ lower relative risk in the respective outcomes by the 2-year time point. Post hoc subgroup analyses from FRAME have suggested that greater efficacy might occur in high-risk patients [7].

Several analyses in a number of placebo-controlled studies have explored the interaction between treatment efficacy and baseline fracture risk characterised using the RRAX $^{\circledR}$ tool [8-15]. This approach avoids the limitations of subgroup analyses by seeking to determine if there is a significant interaction between efficacy and fracture risk, with the latter handled as a continuous variable. Evidence that the efficacy of some interventions is greater in patients with higher baseline FRAX ${ }^{\circledR}$ fracture probabilities has been reported for clodronate [9], bazedoxifene [8], and denosumab [13], but not for other agents such as raloxifene [10], strontium ranelate [11], teriparatide [14], and abaloparatide [16]. We wished to test the clinical hypothesis that there would be an interaction between the efficacy of romosozumab and fracture probabilities assessed at study entry. Given the design of the romosozumab trials described above, the analysis was confined to the first year of the FRAME study.

\section{Methods}

In this independent post hoc analysis of the phase 3 FRAME trial, we used prospectively collected baseline FRAX ${ }^{\circledR}$ risk variables to evaluate the efficacy of romosozumab on fracture outcomes as a continuous function of baseline fracture probability.

Details of the FRAME study are published elsewhere [3]. Briefly, in this international, double-blind, placebo-controlled, parallel group trial, women with postmenopausal osteoporosis were randomly assigned to receive romosozumab in a blinded fashion, at a dose of $210 \mathrm{mg}$, or placebo. Randomization was stratified according to age ( $<75$ years vs. $\geq 75$ years) and prevalent vertebral fracture (yes vs. no). Romosozumab or placebo was administered s.c once monthly for 12 months, followed by open-label denosumab (Prolia, Amgen), at a dose of $60 \mathrm{mg}$ which was administered s.c every 6 months for an additional 12 months. A total of 7108 women between the ages of 55 and 90 years with a BMD T-score less than -2.5 but at least -4.0 at the lumbar spine or total hip were studied; all received daily supplements containing at least $1000 \mathrm{mg}$ of calcium daily with vitamin D (400 to 800 IU daily). Fully anonymised, individual level study data were provided by Amgen to the Centre for Metabolic Bone Diseases, University of Sheffield.

\section{Baseline FRAX probabilities}

Ten-year fracture probability was assessed with the FRAX ${ }^{\circledR}$ tool (version 3.11) in all women at baseline. In brief, clinical risk factors were used to estimate 10 -year probability of a major osteoporotic fracture (MOF: hip, clinical spine, forearm, or humerus fracture) or of a hip fracture alone [17]. The fracture probability can be calculated with clinical risk factors alone, or with femoral neck BMD included. The clinical risk factors comprised sex, age, body mass index (BMI), and dichotomised risk variables documenting a prior fragility fracture, parental history of hip fracture, current tobacco smoking, ever long-term use of oral glucocorticoids, rheumatoid arthritis, other causes of secondary osteoporosis, and alcohol consumption of 3 or more units daily.

For the purposes of this analysis, a prior fragility fracture excluded pathological fractures, any fractures arising before the age of 18 years and fractures at sites not considered to be osteoporotic (i.e. ankle, facial bones, fingers, foot, hand, knee cap, knee, skull, and toes) [18]. One or more prevalent vertebral fractures (grades 1-3 on the Genant scale) on baseline spine imaging were included as a prior fracture.

In addition to the dependence on clinical risk factors, fracture probability varies markedly in different regions of the world $[19,20]$ so that specific ethnic and country models were used as appropriate. For two countries where FRAX ${ }^{\circledR}$ models were not available (Dominican Republic and Latvia), appropriate surrogate models were used (Colombia and Estonia, respectively).

\section{Fracture outcomes}

All incident fractures during the study follow-up were adjudicated and confirmed via x-ray or radiology reports. Sites of fracture, but not ICD codes, were provided and clinical fracture outcomes, regardless of the level of trauma, were allocated to four categories: any clinical fracture (all fractures including those of the feet, ankles, hands, face, and skull); any osteoporotic fracture (as any clinical fracture but excluding those of the feet, ankles, hands, face, and skull); MOF; and hip fracture. Incident vertebral fractures were documented either 
as a clinical vertebral fracture (identified in the presence of back pain and usually requiring radiographic assessment outside of the prespecified schedule) or a morphometric vertebral fracture. The latter were assumed to have occurred mid-way between the date of the scheduled radiograph where the fracture was found and the date of a previous scheduled radiograph, an assumption that does not impact materially on the analysis of efficacy.

\section{Statistical analysis}

This was an intention-to-treat (ITT) analysis. A Poisson model was used to study the relationship between age, the time since baseline (months), treatment and calculated 10-year probability of a major osteoporotic fracture, and their effects on the risk of fracture with only the first of any particular fracture outcome of interest being counted per patient [21, 22]. The extended Poisson regression analysis can estimate effects that vary in time, either linearly or nonlinearly, by studying the interactions between the time in study and the variable of interest (i.e. fracture risk). When there is no interaction with time, this extended Poisson regression model gives results very similar to a Cox regression model. Person-years were used (in contrast to a linear logistic model). The hazard function for fracture was assumed to be:

$\exp \left(\beta_{0}+\beta_{1}\right.$ current time from baseline $+\beta_{2}$ current age + $\beta_{3} 10$-year probability $+\beta_{4}$ treatment $+\beta_{5} 10$ - year probability treatment).

The variable "10-year probability treatment" tests for an interaction between efficacy and baseline 10 -year probability and was handled as a continuous variable by determining if $\beta_{5}$ $>0$. Treatment interactions considered the following fracture outcomes: all clinical fractures, osteoporotic fractures, MOF, clinical vertebral fractures, and morphometric vertebral fractures. A further analysis considered the composite fracture outcomes (e.g. any clinical fracture, osteoporotic fractures, and MOF) with the exclusion of vertebral fractures, thus providing variable representing nonvertebral fractures.

The 10-year probability of a major osteoporotic fracture without the inclusion of BMD was used as the base case in the treatment interaction analyses. Interactions other than 10year probability treatment, such as BMD T-score treatment, previous fracture treatment, and age treatment, were explored in sensitivity analysis. A two-sided $p$ value of 0.1 for the interaction between treatment and FRAX ${ }^{\circledR}$ was considered significant. Additional exploratory analyses included those where hip fracture or nonvertebral fracture was the outcome of interest; where FRAX ${ }^{\circledR}$ was calculated with the inclusion of BMD; and finally, an examination of the effects on the any fracture outcome comparing the Latin American region study centres to the whole study population. The latter was prompted by a post hoc analysis of FRAME that showed that the risk of nonvertebral fracture in the region of Latin America was higher in the romosozumab group than in the placebo group, albeit not significantly (hazard ratio $[\mathrm{HR}]=1.25$; 95\% confidence interval [CI], 0.68-2.27) [3, 7]. By contrast, among the patients outside the region of Latin America, romosozumab significantly decreased the incidence of nonvertebral fracture ( $\mathrm{HR}=0.58 ; 95 \% \mathrm{CI}, 0.37-0.89)[3,7]$.

Hazard ratios for treatment effect and $95 \%$ CI were computed as a continuous variable. For tabular presentation, HRs are shown at the 10th, 25th, 50th, 75th, and 90th percentile of fracture probability. In figures, the associations are shown as a continuous function between the 1st and 99th percentiles except where indicated.

\section{Results}

A summary description of the data used in this FRAX ${ }^{\circledR}$ analysis is provided in Table 1. Data on the clinical risk factors used in FRAX ${ }^{\circledR}$ were available in $7163(99.7 \%)$ of the women included in FRAME. The mean probability of a major osteoporotic fracture was $9.7 \%$ and for a hip fracture was 3.9\% for the base case predictor of FRAX ${ }^{\circledR}$ calculated without BMD. When BMD was used in the FRAX ${ }^{\circledR}$ calculation, the probabilities tended to be greater than those based on clinical risk factors alone, reflecting the need for a relatively low BMD as an inclusion criterion for the trial.

During the first year of FRAME, a total of 178 (2.5\%) women sustained one or more clinical fractures, with $131(1.8 \%)$ and $89(1.2 \%)$ experiencing at least one

Table 1 Summary description of the baseline data provided for calculation of FRAX ${ }^{\circledR}$ probabilities $(n=7163)$

\begin{tabular}{ll}
\hline FRAX $^{\circledR}$ variable & \\
Age $($ years, mean $\pm \mathrm{SD})$ & $70.9 \pm 6.9$ \\
BMI $\left(\mathrm{kg} / \mathrm{m}^{2}\right.$, mean $\left.\pm \mathrm{SD}\right)$ & $24.7 \pm 4.4$ \\
Previous fracture $(\%, n)$ & $18 \%, 1313$ \\
Parental hip fracture $(\%, n)$ & $11 \%, 756$ \\
Current smoking $(\%, n)$ & $10 \%, 745$ \\
Glucocorticoids $(\%, n)$ & $0 \%, 13$ \\
Rheumatoid arthritis $(\%, n)$ & $0 \%, 10$ \\
Secondary osteoporosis $(\%, n)$ & $1 \%, 71$ \\
Alcohol 3 or more units per day $(\%, n)$ & $1 \%, 70$ \\
Femoral neck BMD T-score $($ mean $\pm \mathrm{SD})$ & $-2.80 \pm 0.30$
\end{tabular}

\section{FRAX 10-year probabilities}

$\begin{array}{ll}\text { Hip fracture (including BMD) (mean } \pm \mathrm{SD}) & 5.5 \pm 5.0 \\ \text { Hip fracture (excluding BMD) (mean } \pm \mathrm{SD}) & 3.9 \pm 4.6 \\ \text { MOF (including BMD) (mean } \pm \mathrm{SD}) & 12.5 \pm 8.0 \\ \text { MOF (excluding BMD) (mean } \pm \mathrm{SD}) & 9.7 \pm 7.2\end{array}$

$B M D$, bone mineral density; $B M I$, body mass index; $M O F$, major osteoporotic fracture; $S D$, standard deviation 
osteoporotic fracture or MOF, respectively. Seventy-five $(1.0 \%)$ women sustained one or more new morphometric vertebral fractures, but only $12(0.2 \%)$ sustained clinical vertebral fractures; $22(0.3 \%)$ women sustained a hip fracture.

\section{Overall effects of treatment}

The efficacy of romosozumab on fracture outcomes was observed within the first year of the study when the treatment was compared with placebo (Table 2). Romosozumab significantly decreased the risk of osteoporotic fracture, clinical vertebral fracture, and morphometric vertebral fracture, with reductions in the other fracture outcomes fell short of statistical significance.

\section{Treatment interaction}

There was no significant interaction between any of the variables and time. There was a significant interaction between romosozumab antifracture efficacy and the outcomes of any clinical fracture, osteoporotic fracture, and MOF (Table 3) as shown by a decrease in HRs (i.e. greater efficacy) with increasing fracture probability at baseline. For example, the reduction in any clinical fracture was $19 \%$ with the $95 \%$ CIs embracing unity at the 10th percentile (FRAX ${ }^{\circledR}$ MOF probability $3.7 \%$ ), whereas at the 50th percentile $\left(\right.$ FRAX $^{\circledR}$ MOF probability $7.4 \%$ ), the reduction was $29 \%$ (95\% CI $3-49 \%$ ) (Table 3$)$. At the 90 th percentile $\left(\right.$ FRAX $^{\circledR}$ MOF probability $18.9 \%$ ), the reduction was $54 \%$. The effects of romosozumab for the three categories of fracture outcomes are shown as a continuous function in Fig. 1. For each, there was greater efficacy at higher baseline probabilities. In the case of any clinical fracture, treatment with romosozumab was associated with a significant reduction in fracture risk with probabilities of $7 \%$ or more. For the outcome of osteoporotic fracture and MOF, significant effects of treatment were observed with probabilities of $9 \%$ or more and $12 \%$ or more, respectively. The interactions were no longer significant $(p=0.11-0.21)$ for these outcomes when FRAX $^{\circledR}$ was calculated with bone mineral density (BMD) (data not shown), but the trend was very similar in that higher fracture probabilities were associated with greater efficacy.

In contrast to the interaction between baseline probability and fracture outcomes that were largely composed of fractures at nonvertebral sites, the HRs were stable across the range of baseline FRAX ${ }^{\circledR}$ probabilities for vertebral fracture, both morphometric and clinical fracture (Table 3), consistent with similar treatment efficacy regardless of baseline risk. As expected, when clinical vertebral fractures were excluded from the outcomes of any clinical fracture, osteoporotic fracture, and MOF, the significance of the interactions between romosozumab efficacy and baseline fracture probabilities became even more marked $(p=0.046, p=0.046$, and $p=0.039$, respectively). This is illustrated for osteoporotic fractures without vertebral fractures (i.e. nonvertebral fractures) in Table 3 and Fig. 1. Given the relatively small number of incident hip fractures in the first year of FRAME, no significant interaction was seen for this outcome, though a similar trend was observed to that at overall nonvertebral sites.

In the additional analyses looking at potential interaction between romosozumab efficacy and individual risk factors, there were no statistically significant interactions with the continuous (age, BMI) or categorical (smoking, prior fracture, and parental history) baseline variables. An exception was an unexpected interaction between baseline femoral neck BMD and efficacy $(p=0.097)$ in the sense that the lower the T-score, the less the effect. The differences in efficacy at different $\mathrm{T}$-scores were, however, modest. For example, at a T-score of - 2.99 the HR for clinical fractures was 0.54 (95\% CI 0.38-0.76). At a T-score of -2.62 , the HR was 0.41 (95\% CI $0.28-0.59$ ).

\section{Regional variation}

The baseline median fracture probabilities were lower in the Latin American centres compared with other regions for MOF (5.0\% vs. $10.0 \%$, respectively, calculated without BMD) and hip fracture (1.9\% vs. $3.2 \%$, respectively). The result of the interaction analysis for the outcome of any clinical fracture, confined to the Latin American centres, is shown superimposed on the overall study analysis in Fig. 2. The data from the Latin American countries showed a similar
Table 2 Effects of romosozumab compared to placebo over 12 months according to the fracture outcome. The current analysis used a Poisson model adjusting for age and time since baseline

\begin{tabular}{lll}
\hline & Treatment effect (HR, 95\% CI) & Two-sided $p$ value \\
\hline Any fracture & $0.63(0.47,0.85)$ & 0.003 \\
Osteoporotic fracture & $0.66(0.46,0.93)$ & 0.018 \\
Major osteoporotic fracture & $0.68(0.44,1.03)$ & 0.071 \\
Hip fractures & $0.47(0.19,1.14)$ & 0.096 \\
Nonvertebral fracture & $0.72(0.50,1.03)$ & 0.071 \\
Clinical vertebral fracture & $0.20(0.04,0.91)$ & 0.038 \\
Morphometric vertebral fracture & $0.27(0.16,0.47)$ & $<0.001$ \\
\hline
\end{tabular}


Table 3 Hazard ratio between treatments (romosozumab versus placebo) in the first year of FRAME for various fracture outcomes at different values of 10-year probability (\%) of a MOF calculated without BMD. Italicised numbers indicate that the $95 \%$ CIs for the effect estimate exclude unity

\begin{tabular}{llllllll}
\hline Percentile & $\begin{array}{l}\text { 10-year } \\
\text { probability }(\%)\end{array}$ & $\begin{array}{l}\text { Any clinical } \\
\text { fracture }\end{array}$ & Osteoporotic fracture & $\begin{array}{l}\text { Major osteoporotic } \\
\text { fracture }\end{array}$ & $\begin{array}{l}\text { Nonvertebral } \\
\text { fracture }\end{array}$ & $\begin{array}{l}\text { Morphometric } \\
\text { vertebral fracture }\end{array}$ & $\begin{array}{l}\text { Clinical vertebral } \\
\text { fracture }\end{array}$ \\
\hline 10 th & 3.65 & $0.81(0.55-1.20)$ & $0.88(0.56-1.40)$ & $0.96(0.55-1.68)$ & $1.00(0.62-1.61)$ & $0.25(0.12-0.52)$ & $0.14(0.02-1.10)$ \\
25 th & 4.70 & $0.78(0.54-1.13)$ & $0.84(0.55-1.30)$ & $0.91(0.54-1.54)$ & $0.95(0.61-1.49)$ & $0.26(0.13-0.51)$ & $0.15(0.02-1.04)$ \\
50 th & 7.38 & $0.71(0.51-0.97)$ & $0.76(0.52-1.10)$ & $0.80(0.51-1.27)$ & $0.84(0.57-1.24)$ & $0.26(0.14-0.48)$ & $0.17(0.03-0.93)$ \\
75 th & 12.23 & $0.59(0.43-0.81)$ & $0.62(0.43-0.89)$ & $0.64(0.41-0.99)$ & $0.67(0.46-0.98)$ & $0.27(0.16-0.47)$ & $0.21(0.04-0.98)$ \\
90 th & 18.88 & $0.46(0.29-0.73)$ & $0.47(0.28-0.79)$ & $0.47(0.25-0.87)$ & $0.49(0.29-0.84)$ & $0.29(0.16-0.52)$ & $0.29(0.04-1.93)$ \\
$\begin{array}{l}p \text { value for } \\
\text { interaction* }\end{array}$ & 0.06 & 0.07 & 0.08 & 0.046 & $>0.30$ & $>0.30$ \\
\hline
\end{tabular}

*Two-sided $p$ value for interaction between treatment and FRAX ${ }^{\circledR}$

${ }^{a}$ Derived by excluding vertebral fractures from osteoporotic fractures

qualitative relationship between efficacy and FRAX ${ }^{\circledR}$, though this fell short of statistical significance $(p>0.30)$ with wide confidence limits. Similar findings were observed for the outcome of osteoporotic fracture (data not shown).

\section{Discussion}

The aim of this analysis was to determine the interaction, if any, between baseline fracture probability and the efficacy of romosozumab to allow comparison with similar analyses from placebo-controlled trials of other osteoporosis treatments. The design of the phase 3 trials of romosozumab posed challenges
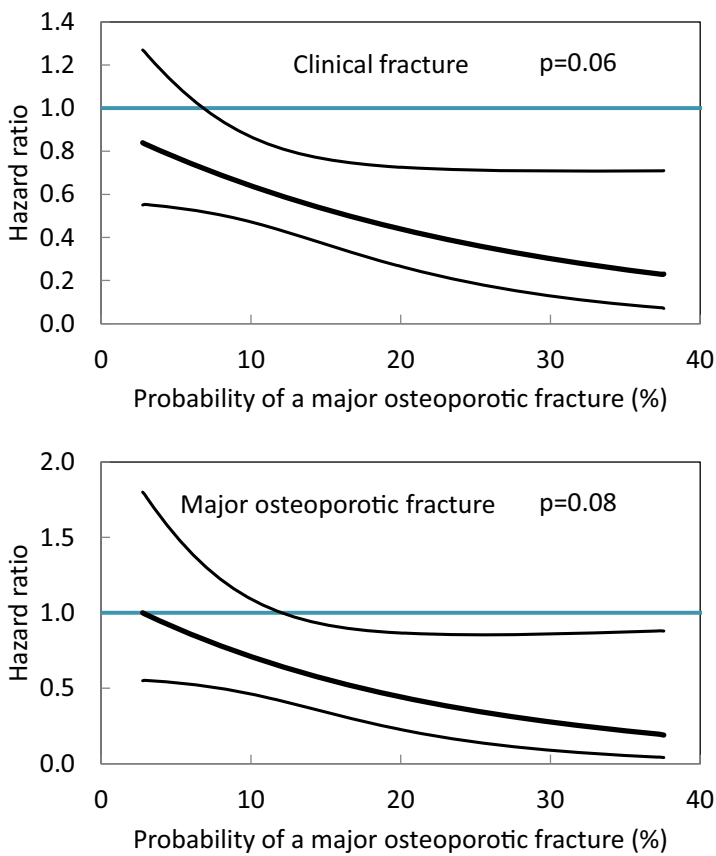

Fig. 1 Effect of romosozumab on any clinical fracture (top left panel), osteoporotic fracture (top right panel), MOF (lower left panel), and nonvertebral fracture (osteoporotic fractures excluding vertebral to fulfil this aim in that only the first placebo-controlled year of the FRAME study provided the opportunity for such an assessment. The category of "any clinical fracture" (which included fractures of the hand, feet, face, and skull) was added in this analysis to ensure that the power of the study was optimised by accounting for all fractures occurring within that 1 -year period. While this study's approach to the analysis was not identical to that used in the original publications of the FRAME trial, it is important to note that this study's results on overall fracture risk reductions in the first year were very comparable to those reported previously [3, 23]. Importantly, the present analysis also demonstrates significant interactions between efficacy and baseline FRAX fracture probability,
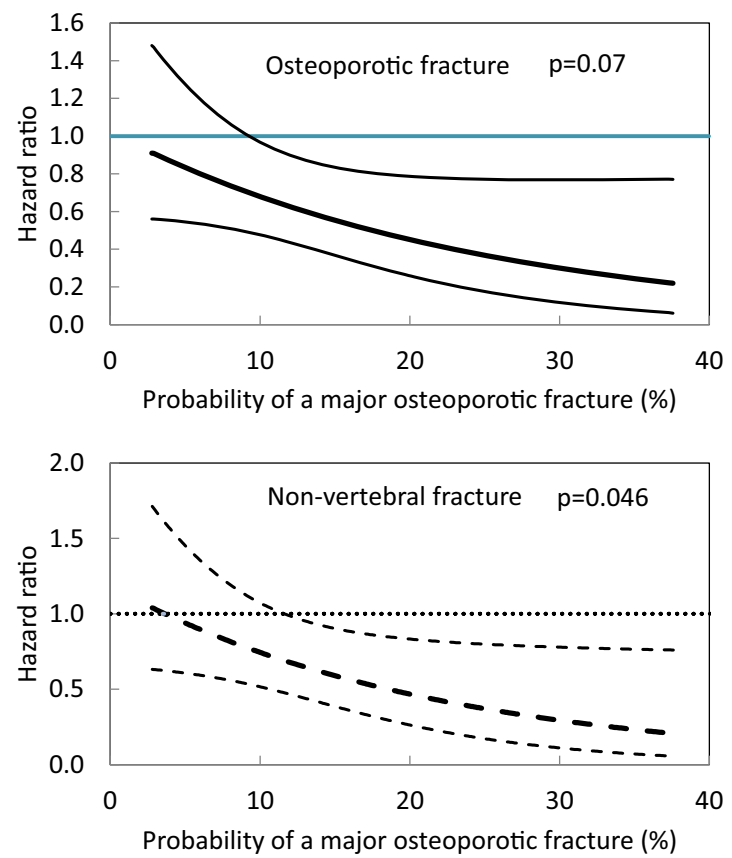

fractures) (lower right panel) compared with placebo expressed as HR with $95 \%$ CIs across the range of MOF probabilities at baseline calculated without BMD 
Fig. 2 Effect of romosozumab on any clinical fracture compared with placebo expressed as the HR with $95 \%$ CIs across the range of MOF probabilities (1st to 99 th percentile) at baseline calculated without BMD. Dotted lines are derived from the first year of the FRAME study. Solid lines depict the first-year data from the Latin American countries

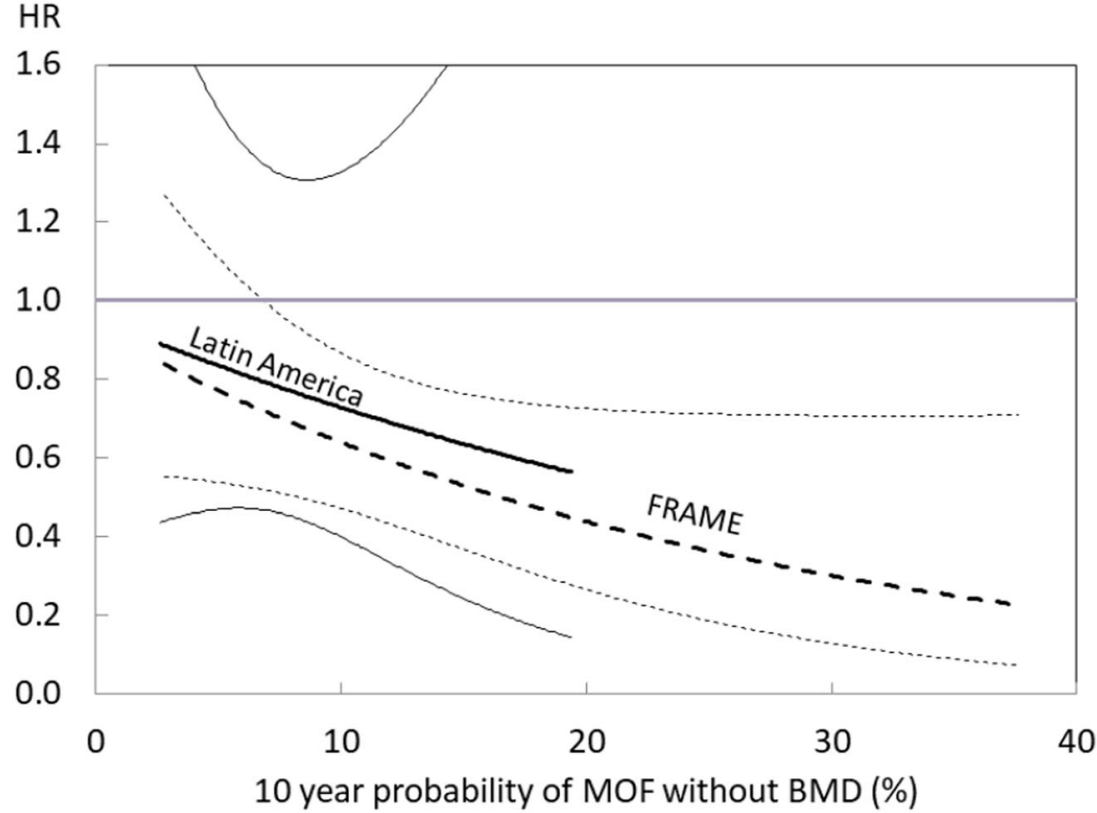

calculated without BMD. Hazard ratios decreased (i.e. greater efficacy) with increasing fracture probability for any clinical fracture, osteoporotic fracture, and MOF, suggesting that the benefits of romosozumab will be most marked in patients at high risk of fracture. Notably, a similar pattern was observed when BMD was included in the calculation of FRAX ${ }^{\circledR}$ probabilities, though this did not reach statistical significance. The reasons for this are not clear, but as reported, we observed an unexpected relationship between femoral neck BMD with lower romosozumab efficacy at lower BMD. It is possible that the relationship between the risk of fracture and BMD was not as expected, given that low BMD was an inclusion criterion for the trial but associated risk factors such as prior hip and moderate/severe vertebral fractures were exclusion criteria, and this may have introduced confounding. In addition, the inclusion of patients with low BMD from countries with low risk of fracture might also have contributed to this observation.

The observation that the interaction is only observed for nonvertebral fractures, and not for vertebral fractures, appears to be a novel finding. It has been appreciated for many years that the responsiveness to osteoporosis treatments varies between vertebral and nonvertebral sites; certainly, the size and speed of onset of effect of treatment have frequently been observed as greater for vertebral than nonvertebral fracture. In the current study, the majority of analyses of the effects of treatment-induced reductions in vertebral fractures across the range of baseline FRAX ${ }^{\circledR}$ probability revealed no significant interactions; the similar and stable efficacy suggests a floor/ceiling effect for each drug on vertebral fracture outcomes. For example, in the setting of anabolic therapy, teriparatide administered daily or weekly has shown reductions in vertebral fractures of 66-79\% without any interaction with baseline fracture risk $[14,15]$. The interaction with baseline probability for nonvertebral fractures seen with romosozumab raises questions about the mechanism of fracture reductions in the setting of increased fracture risk.

Several studies have examined the interaction between FRAX $^{\circledR}$-based probabilities with effectiveness in placebocontrolled studies, including treatment with raloxifene [10], bazedoxifene [8], clodronate [9], denosumab [13], alendronate [12], daily and weekly teriparatide $[14,15]$, and abaloparatide [16]. Several of these studies have shown greater efficacy against fracture in individuals at higher risk when treated with clodronate, bazedoxifene, or denosumabromosozumab can now be added. This FRAX ${ }^{\circledR}$ dependency has marked consequences for health economic analysis, illustrated when comparing the cost-effectiveness of the two selective oestrogen receptor modulators, raloxifene and bazedoxifene [24]. The appreciation of greater efficacy at higher baseline risks, as seen with bazedoxifene, can alter conclusions drawn about cost-effectiveness, with more expensive but more effective agents potentially preferred in those at highest risk of fracture. Superior and more rapid antifracture efficacy has now been shown for anabolic treatments in headto-head trials with antiresorptive agents, including teriparatide compared with risedronate [25] and romosozumab compared with alendronate [4]. This knowledge is starting to influence clinical guidelines and approaches to management, where there is increasing recognition that anabolic agents might be seen as first-line agents in patients at very high risk of fracture, especially where the risk might be particularly high in the short term, for example after a recent osteoporotic fracture $[26,27]$. 
These findings are helpful in explaining apparent regional differences in the efficacy of romosozumab on nonvertebral fracture [3]. The FRAME study reported that romosozumab did not significantly decrease the incidence of nonvertebral fractures in the first year $(\mathrm{HR}=0.75 ; 95 \%$ CI $0.53-1.05)$ with a similar effect size at the end of the second year, when both groups had received denosumab for a year. A subsequent post hoc analysis showed that the effect of romosozumab on nonvertebral fractures differed between the Latin American region $(\mathrm{HR}=1.25 ; 95 \% \mathrm{CI} 0.68-2.27)$ and other regions $(\mathrm{HR}=0.58 ; 95 \%$ CI $0.37-0.89)[3,7]$. The present findings indicate that apparent regional differences in efficacy are likely related to differences in fracture probability rather than to ethnicities or other reasons. This view is supported by the observation in the present analysis that high-risk patients in Latin America appear to respond to treatment in much the same way as in the FRAME cohort in general. A caveat is that the interaction term between efficacy and FRAX ${ }^{\circledR}$ was not significant in the analysis confined to women from Latin America, but this is limited by the low number of events in this population.

This analysis has a number of strengths and limitations. Subgroup analyses, especially those undertaken post hoc, reduce the statistical power of studies and increase the risk of type 1 errors [28]. Examining for interactions using a continuous risk variable, such as baseline fracture probability, reduces the erroneous impact of categorical subgroup analyses. For example, in a post hoc analysis of romosozumab efficacy on nonvertebral fracture risk using an intervention threshold in keeping with US treatment thresholds (FRAX ${ }^{\circledR} 10$-year probability of major osteoporotic fracture $\geq 20 \%$ or hip fracture $\geq$ $3 \%$ ) suggested that romosozumab was ineffective below this threshold [7]. The current analysis suggests that efficacy of romosozumab can be observed at substantially lower thresholds, for example at MOF probabilities of 9$12 \%$ for the outcomes of osteoporotic or major osteoporotic fractures. The present analysis is confined to a total treatment exposure of just 1 year, a substantially shorter duration than in other placebo-controlled studies in osteoporosis. Nonetheless, the rapid efficacy of romosozumab on vertebral and clinical fracture outcomes delivers an efficacy that can then be examined for interactions.

In conclusion, there are significant interactions between baseline fracture probability and the effect of romosozumab on clinical fracture, osteoporotic fracture, and MOF when compared with placebo in the first year of the FRAME study. Furthermore, the evidence supports the view that high-risk patients in Latin America appear to respond to treatment in much the same way as in the FRAME cohort in general. The increased efficacy of romosozumab at higher levels of baseline risk should be taken into account in health economic analyses.
Acknowledgments Alexandra Stirling of BioScience Communications, New York, NY (on behalf of Amgen, Inc.), provided editorial support.

Funding This study was funded by Amgen, Inc., Astellas Pharma Inc., and UCB.

Data availability Qualified researchers may request data from Amgen clinical studies. Complete details are available at the following: https:// wwwext.amgen.com/science/clinical-trials/clinical-data-transparencypractices/clinical-trial-data-sharing-request/.

\section{Compliance with ethical standards}

Conflicts of interest Eugene V McCloskey has received consultancy/ lecture fees/grant funding/honoraria from ActiveSignal, AgNovos, Amgen, AstraZeneca, Consilient Healthcare, Fresenius Kabi, Gilead, GSK, Hologic, Internis, Lilly, Medtronic, Merck, Novartis, Pfizer, Radius Health, Redx Oncology, Roche, SanofiAventis, Servier, Synexus, Tethys, UCB, Viiv, Warner Chilcott, I3 Innovus, and Unilever, all outside the presented work. Helena Johansson has no conflicts to declare. Nicholas C Harvey has received consultancy/lecture fees/honoraria/grant funding from Alliance for Better Bone Health, Amgen, MSD, Eli Lilly, Radius Health, Servier, Shire, UCB, Consilient Healthcare, and Internis Pharma, all outside the presented work. Mattias Lorentzon has received lecture fees from Amgen, Lilly, Meda, Renapharma, and UCB Pharma, and consulting fees from Amgen, Radius Health, UCB Pharma, Renapharma, and Consilient Health, all outside the presented work. Yifei Shi is an employee and stockholder of Amgen Inc. John A Kanis reports grants from Amgen, Eli Lilly, and Radius Health and consulting fees from Theramex. He led the team that developed FRAX ${ }^{\circledR}$ as director of the then WHO Collaborating Centre for Metabolic Bone Diseases; he has no financial interest in FRAX ${ }^{\circledR}$.

Ethical approval All procedures performed in studies involving human participants were in accordance with the ethical standards of the institutional and/or national research committee and with the 1964 Helsinki declaration and its later amendments or comparable ethical standards.

Consent to participate For this type of study, formal consent is not required.

Consent for publication Not applicable.

Statement of human rights The manuscript does not contain clinical studies or patient data. For this type of study, formal consent is not required.

Code availability Not applicable.

Open Access This article is licensed under a Creative Commons Attribution-NonCommercial 4.0 International License, which permits any non-commercial use, sharing, adaptation, distribution and reproduction in any medium or format, as long as you give appropriate credit to the original author(s) and the source, provide a link to the Creative Commons licence, and indicate if changes were made. The images or other third party material in this article are included in the article's Creative Commons licence, unless indicated otherwise in a credit line to the material. If material is not included in the article's Creative Commons licence and your intended use is not permitted by statutory regulation or exceeds the permitted use, you will need to obtain permission directly from the copyright holder. To view a copy of this licence, visit http:// creativecommons.org/licenses/by-nc/4.0/. 


\section{References}

1. McClung MR, Grauer A, Boonen S et al (2014) Romosozumab in postmenopausal women with low bone mineral density. N Engl J Med 370:412-420

2. Padhi D, Jang G, Stouch B, Fang L, Posvar E (2011) Single-dose, placebo-controlled, randomized study of AMG 785, a sclerostin monoclonal antibody. J Bone Miner Res 26:19-26

3. Cosman F, Crittenden DB, Adachi JD, Binkley N, Czerwinski E, Ferrari S, Hofbauer LC, Lau E, Lewiecki EM, Miyauchi A, Zerbini CAF, Milmont CE, Chen L, Maddox J, Meisner PD, Libanati C, Grauer A (2016) Romosozumab treatment in postmenopausal women with osteoporosis. N Engl J Med 375:1532-1543

4. Saag KG, Petersen J, Brandi ML, Karaplis AC, Lorentzon M, Thomas T, Maddox J, Fan M, Meisner PD, Grauer A (2017) Romosozumab or alendronate for fracture prevention in women with osteoporosis. N Engl J Med 377:1417-1427

5. Cosman F, Crittenden DB, Ferrari S, Khan A, Lane NE, Lippuner K, Matsumoto T, Milmont CE, Libanati C, Grauer A (2018) FRAME study: the foundation effect of building bone with 1 year of romosozumab leads to continued lower fracture risk after transition to denosumab. J Bone Miner Res 33:1219-1226

6. Lewiecki EM, Dinavahi RV, Lazaretti-Castro M, Ebeling PR, Adachi JD, Miyauchi A, Gielen E, Milmont CE, Libanati C, Grauer A (2019) One year of romosozumab followed by two years of denosumab maintains fracture risk reductions: results of the FRAME extension study. J Bone Miner Res 34:419-428

7. Cosman F, Crittenden DB, Ferrari S, Lewiecki EM, Jaller-Raad J, Zerbini C, Milmont CE, Meisner PD, Libanati C, Grauer A (2018) Romosozumab FRAME study: a post hoc analysis of the role of regional background fracture risk on nonvertebral fracture outcome. J Bone Miner Res 33:1407-1416

8. Kanis JA, Johansson H, Oden A, McCloskey EV (2009) Bazedoxifene reduces vertebral and clinical fractures in postmenopausal women at high risk assessed with FRAX. Bone 44:10491054

9. McCloskey EV, Johansson H, Oden A, Vasireddy S, Kayan K, Pande K, Jalava T, Kanis JA (2009) Ten-year fracture probability identifies women who will benefit from clodronate therapyadditional results from a double-blind, placebo-controlled randomised study. Osteoporos Int 20:811-817

10. Kanis JA, Johansson H, Oden A, McCloskey EV (2010) A metaanalysis of the efficacy of raloxifene on all clinical and vertebral fractures and its dependency on FRAX. Bone 47:729-735

11. Kanis JA, Johansson H, Oden A, McCloskey EV (2011) A metaanalysis of the effect of strontium ranelate on the risk of vertebral and non-vertebral fracture in postmenopausal osteoporosis and the interaction with FRAX®. Osteoporos Int 22:2347-2355

12. Donaldson MG, Palermo L, Ensrud KE, Hochberg MC, Schousboe JT, Cummings SR (2012) Effect of alendronate for reducing fracture by FRAX score and femoral neck bone mineral density: the Fracture Intervention Trial. J Bone Miner Res 27:1804-1810

13. McCloskey EV, Johansson H, Oden A, Austin M, Siris E, Wang A, Lewiecki EM, Lorenc R, Libanati C, Kanis JA (2012) Denosumab reduces the risk of osteoporotic fractures in postmenopausal women, particularly in those with moderate to high fracture risk as assessed with FRAX. J Bone Miner Res 27:1480-1486

14. Harvey NC, Kanis JA, Oden A, Burge RT, Mitlak BH, Johansson H, McCloskey EV (2015) FRAX and the effect of teriparatide on vertebral and non-vertebral fracture. Osteoporos Int 26:2677-2684

15. Harvey NC, Kanis JA, Oden A, Nakamura T, Shiraki M, Sugimoto T, Kuroda T, Johansson H, McCloskey EV (2015) Efficacy of weekly teriparatide does not vary by baseline fracture probability calculated using FRAX. Osteoporos Int 26:2347-2353
16. McCloskey EV, Johansson H, Oden A, Harvey NC, Jiang H, Modin S, Fitzpatrick L, Kanis JA (2017) The effect of abaloparatide-SC on fracture risk is independent of baseline FRAX fracture probability: a post hoc analysis of the ACTIVE study. J Bone Miner Res 32:1625-1631

17. Kanis JA on behalf of the World Health Organization Scientific Group (2007) Assessment of osteoporosis at the primary healthcare level. Technical Report. World Health Organization Collaborating Centre for Metabolic Bone Diseases, University of Sheffield, UK. 2007

18. Kanis JA, Oden A, Johnell O, Jonsson B, de Laet C, Dawson A (2001) The burden of osteoporotic fractures: a method for setting intervention thresholds. Osteoporos Int 12:417-427

19. Kanis JA, Johnell O, De Laet C, Jonsson B, Oden A, Ogelsby AK (2002) International variations in hip fracture probabilities: implications for risk assessment. J Bone Miner Res 17:1237-1244

20. Kanis JA, Oden A, EV MC, Johansson H, Wahl DA, Cooper C, for the IOF Working Group on Epidemiology and Quality of Life (2012) A systematic review of hip fracture incidence and probability of fracture worldwide. Osteoporos Int 23:2239-2256

21. Breslow NE, Day NE (1987) Statistical methods in cancer research. IARC Sci Publ No 32 II:131-135

22. Albertsson-Wikland K, Martensson A, Savendahl L et al (2016) Mortality is not increased in recombinant human growth hormone-treated patients when adjusting for birth characteristics. J Clin Endocrinol Metab 101:2149-2159

23. Geusens P, Oates M, Miyauchi A, Adachi JD, Lazaretti-Castro M, Ebeling PR, Perez Nino CA, Milmont CE, Grauer A, Libanati C (2019) The effect of 1 year of romosozumab on the incidence of clinical vertebral fractures in postmenopausal women with osteoporosis: results from the FRAME study. JBMR Plus 3:e10211. https://doi.org/10.1002/jbm4.10211

24. Kim K, Svedbom A, Luo X, Sutradhar S, Kanis JA (2014) Comparative cost-effectiveness of bazedoxifene and raloxifene in the treatment of postmenopausal osteoporosis in Europe, using the FRAX algorithm. Osteoporos Int 25:325-337

25. Kendler DL, Marin F, Zerbini CAF, Russo LA, Greenspan SL, Zikan V, Bagur A, Malouf-Sierra J, Lakatos P, FahrleitnerPammer A, Lespessailles E, Minisola S, Body JJ, Geusens P, Möricke R, López-Romero P (2018) Effects of teriparatide and risedronate on new fractures in post-menopausal women with severe osteoporosis (VERO): a multicentre, double-blind, doubledummy, randomised controlled trial. Lancet 391:230-240

26. Khosla S (2019) Personalising osteoporosis treatment for patients at high risk of fracture. Lancet Diabetes Endocrinol 7:739-741

27. Kanis JA, Harvey NC, McCloskey E, Bruyère O, Veronese N, Lorentzon M, Cooper C, Rizzoli R, Adib G, al-Daghri N, Campusano C, Chandran M, Dawson-Hughes B, Javaid K, Jiwa F, Johansson H, Lee JK, Liu E, Messina D, Mkinsi O, Pinto D, Prieto-Alhambra D, Saag K, Xia W, Zakraoui L, Reginster JY (2020) Algorithm for the management of patients at low, high and very high risk of osteoporotic fractures. Osteoporos Int 31:1-12

28. Higgins JPT, Thomas J, Chandler J, Cumpston M, Li T, Page MJ, Welch VA (editors). Cochrane handbook for systematic reviews of interventions version 6.0 (updated July 2019). Cochrane, 2019. Available from www.training.cochrane.org/handbook. Kanis JA, on behalf of the WHO Scientific Group (2008) Assessment of osteoporosis at the primary health-care level. Technical Report. Sheffield, WHO Collaborating Centre, University of Sheffield, UK.

Publisher's note Springer Nature remains neutral with regard to jurisdictional claims in published maps and institutional affiliations. 